\title{
Development of Bing-Neel syndrome Despite Ibrutinib Therapy for Waldenstrom Macroglobulinemia: Next Steps in Management and Review of Future Directions
}

\author{
Lukas Delasos $^{1}$, Deep Phachu ${ }^{1}$, Nishka Shetty ${ }^{1}$, Melissa Sepulveda-Ramos $^{2}$, and James \\ Vredenburgh ${ }^{3}$ \\ ${ }^{1}$ University of Connecticut Health Center \\ ${ }^{2}$ Hartford Hospital Department of Pathology and Laboratory Medicine \\ ${ }^{3}$ Smilow Cancer Hospital at St. Francis
}

May 13, 2020

\begin{abstract}
Bing-Neel syndrome (BNS) remains a rare complication of Waldenstrom Macroglobulinemia. Given the paucity of this disease, treatment guidelines are based on small clinical trials with limited participants. Here we present a case of BNS that developed while on ibrutinib therapy, followed by a review of the next steps in management.
\end{abstract}

Keywords

Waldenstrom Macroglobulinemia, Bing-Neel syndrome, central nervous system lymphomas, hematology, oncology

Key Clinical Message

Even when disease appears stable while on systemic therapy for Waldenstrom Macroglobulinemia, Bing-Neel syndrome can manifest and its early recognition may lead to improved quality of life and progression free survival during these rare instances.

\section{Introduction}

Waldenstrom macroglobulinemia (WM) is a form of lymphoplasmacytic lymphoma characterized by the malignant proliferation of monoclonal immunoglobulin M (IgM)-producing B-lymphocytes resulting in a wide spectrum of complications [1]. WM is a rare disorder with an estimated incidence rate of 3 per $10^{6}$ new cases per year [2]. Although presenting signs and symptoms of this disease can vary greatly from patient to patient, all are related to bone marrow infiltration of lymphoplasmacytic cells as well as the effects from a monoclonal gammopathy causing hyperviscosity, end-organ deposition, and autoimmune disease [1,2]. Clinical manifestations include constitutional symptoms such as fevers, night sweats, and unintentional weight loss as well as symptoms related to anemia and hyperviscosity such as fatigue, dyspnea, and headaches [1-2]. Neurological complications associated with hyperviscosity from WM include visual changes (i.e. blurred vision), vertigo, tinnitus, and peripheral neuropathy which is most commonly characterized by bilateral and symmetrical sensory deficits in the hands and feet that can progress to difficulty writing and gait instability [1-2]. Disease involvement outside of the bone marrow most commonly affects the lymph nodes and spleen, with up to $60 \%$ of patients developing extramedullary disease upon relapse [1]. However, direct invasion of the central nervous system (CNS) with lymphoplasmacytic tumor cells, referred to as Bing-Neel syndrome (BNS), remains an extremely rare complication of WM affecting less than $1 \%$ of cases [2,3]. Bing-Neel 
syndrome usually presents as a feature of relapsing disease and should be suspected when there is the onset of central neurological deficits such as altered mentation, cranial nerve deficits, seizure-like activity, gait disturbances, or even psychiatric disease [2,3]. Symptoms are usually gradual, progressing over weeks to months, and may be mistaken as hyperviscosity syndrome or neuropathy [3]. Here, we present a case of WM with malignant CNS involvement visualized on MRI with negative CSF studies but confirmed on brain biopsy as BNS.

\section{Case Presentation}

A 70-year-old Caucasian female with medical history significant for stage III chronic kidney disease, transitional cell ureteral cancer status post left-sided nephroureterectomy, and Waldenstrom's macroglobulinemia (WM) presented with complaints of right-sided weakness associated with paresthesias, dysarthria, and blurry vision of three weeks duration. Magnetic resonance (MRI) imaging of the brain demonstrated an enhancing, hypercellular mass centered in the left thalamus with additional foci of signal abnormality and enhancement in the cortex of the left frontal lobe and subcortical white matter [Figure 1]. These findings were concerning for an intracranial neoplastic process, especially given her history of WM.

Regarding her oncological history, she was initially diagnosed with WM at the age of 67 after workup for complaints of chronic fatigue revealed elevated IgM levels $(3370 \mathrm{mg} / \mathrm{dL})$ as well as serum hyperviscosity. Bone marrow biopsy showed a low-grade B-cell lymphoma with plasmacytic differentiation and 60-70\% bone marrow involvement. Neoplastic cells were found to be lambda restricted and negative for CD5, CD10, and CD23 by flow cytometry. An increased number of lambda predominant cells were confirmed by flow cytometry and CD138 immunostaining. The patient was started on first-line therapy with the Bruton tyrosine kinase inhibitor ibrutinib, however, due to worsening adverse effects she transitioned therapy to rituximab, an anti-CD20 monoclonal antibody. Unfortunately, the patient was found to have worsening IgM levels and serum viscosity while on rituximab monotherapy. Thus, she was restarted on ibrutinib while continuing rituximab every 3 months and had significant improvement on this combination of therapy.

She completed two years of maintenance rituximab and reduced-dose ibrutinib (140 $\mathrm{mg})$ at time of presentation with the most recent IgM levels of $299 \mathrm{mg} / \mathrm{dL}$ prior to the onset of her previously mentioned neurological symptoms. Given her MRI findings, computed tomography (CT) imaging of the head, chest, abdomen, and pelvis was completed which revealed multiple intracranial lesions but no evidence of lymphadenopathy or neoplastic process elsewhere. She further underwent lumbar puncture for cerebral spinal fluid (CSF) analysis with flow cytometry showing mostly T-cells without evidence of B-cell non-Hodgkin lymphoma. As there remained high suspicion for central nervous system (CNS) lymphoma, the patient ultimately had a left parietal stereotactic brain biopsy with pathology findings of diffuse aggressive B-cell non-Hodgkin lymphoma [Figure 3A]. Immunohistochemical studies were positive for CD20, CD23, BCL-6, MUM1, and LE1 [Figure 3B] with approximately $80 \%$ of cells expressing Ki-67 proliferation antigen [Figure 3C]. Fluorescent in situ hybridization (FISH) analysis was negative for c-MYC, BCL6, or BCL2 gene rearrangements. Lastly, mutation testing using next-generation sequencing returned positive for MYD88 L265P mutation, suggesting CNS infiltration of the patient's previously known WM (i.e. Bing-Neel syndrome) rather than the development of a primary CNS lymphoma.

The patient was placed on oral dexamethasone $4 \mathrm{mg}$ four times daily with noticeable improvement in her speech and mobility. Due to the patient's poor renal function, she was not a candidate for induction therapy with methotrexate. Thus, she began treatment with whole brain radiation therapy (WBRT) to 30.6 Gy while continuing systemic treatment with ibrutinib. A repeat MRI of the brain two months later demonstrated near resolution of the patient's lymphoma with findings of only a few small foci of nonspecific enhancement adjacent to the biopsy cavity within the left thalamus [Figure 2]. There was no evidence of intracranial mass effect, midline shift, or abnormal extra-axial collection.

\section{Discussion}

Waldenstrom macroglobulinemia (WM) is defined by the World Health Organization (WHO) as an indolent lymphoplasmacytic lymphoma (LPL) belonging to the category of non-Hodgkin B-cell lymphomas (NHL) 
[4]. Although there remains a lack of specific chromosomal or oncogene abnormalities in LPL, $90 \%$ of cases share MYD88 L265P mutations that are most commonly associated with IgM monoclonal gammopathies such as WM [4]. While WM remains incurable, median survival rates have increased from 5 to 8 years due to increased awareness of the disease and advancements in therapy over the last decade [2]. Essential aspects of the initial evaluation for WM include a detailed history and physical examination prior to pursuing diagnostic workup. Although constitutional symptoms of this disease are shared amongst various other lymphoid malignancies, thorough history taking may reveal clinical manifestations specific to hyperviscosity syndrome such as spontaneous epistaxis, recurrent headaches, visual changes, and blurred vision $[1,2]$. Peripherally circulating antibodies not only cause detrimental effects through changes in blood viscosity, but also by their deposition in end-organs and through immune system autoreactivity [5]. However, the multiple complications associated with WM are not simply related to the effects of a monoclonal gammopathy. For example, peripheral neuropathy which is observed in approximately $20 \%$ of patients who suffer from this disease process may be a result of direct lymphoplasmacytic infiltration or IgM deposition of nerve fibers, amyloidosis from excess light chain production, or development of autoimmunity $[1,2]$.

In approximately $1 \%$ of WM cases, patients develop Bing-Neel syndrome (BNS) in which there is infiltration of the CNS by lymphoplasmacytic cells. The absolute incidence of BNS is unknown, but in a retrospective cohort study of $1,523 \mathrm{WM}$ patients, only 13 patients $(0.8 \%)$ were diagnosed with BNS, suggesting a very low prevalence [3,6]. A review of published literature shows primarily case reports and few small retrospective surveys, demonstrating the rarity of this disease manifestation. Retrospective analysis has demonstrated a significantly lower median survival time (4 months) between symptom onset and diagnosis of BNS as compared to WM [3]. To further complicate matters, BNS can present at any time during the active treatment course for WM, even when the patient is in apparent remission from disease [1-3]. Rarely, BNS precedes the diagnosis of WM and appears as a primary CNS LPL, having only been described in twenty-four cases [7]. Prognosis appears to be better in these cases as compared to those with a prior history of WM whose disease progresses to BNS [3].

The evaluation for BNS begins with diagnostic testing including gadolinium-enhanced magnetic resonance imaging (MRI) of the brain and whole spine as well as cerebrospinal fluid (CSF) sampling for cytology, flow cytometry, and mutational analyses [1-3]. Findings on MRI include contrast-enhanced infiltrations with or without thickening of the meningeal sheaths depending on leptomeningeal involvement, in addition to accentuated diffusion weight imaging (DWI) with elevated or normal apparent diffusion coefficient (ADC) values suggestive of vasogenic edema due to perivascular space invasion by malignant cells [9]. Of note, there are two categories of BNS that can be distinguished on MRI: a diffuse form, in which there is leptomeningeal and perivascular infiltration versus a tumoral form which is unifocal or multifocal and usually involves the deep subcortical hemispheric regions [3, 9]. Infrequently, infraorbital or periorbital involvement can also be seen [20]. In regards to our patient, she presented as the tumoral form of BNS with evidence of multiple enhancing lesions within the subcortical white matter. Although these different forms of BNS from MRI findings are described extensively in literature, actual invasion of malignant lymphoplasmacytic cells may be more extensive than what is evident on imaging. Autopsy reports are scarce given the rarity of this disease, but there appears to be prominent perivascular infiltration by malignant cells without deposition of IgM in BNS that is not readily apparent on MRI imaging [10].

Despite these sensitive imaging techniques, MRI cannot distinguish between other forms of CNS lymphoma thus necessitating further testing with both CSF analysis and tissue biopsy. CSF should be collected and sent for cytology, flow cytometry, in addition to electrophoresis and immunofixation in order to determine whether M-protein and specific immunoglobulin elevations are present within the CNS [1-3]. Yet, results from immunofixation may be skewed by other form of lymphoplasmacytic lymphomas. Thus, mutational analysis of CSF plays a prominent role in the workup of BNS. As is seen in approximately 90-97\% of WM cases, MYD88 L265P gene mutation identified by either next generation sequencing (NGS) or real-time quantitative PCR (qPCR) aids in diagnosis of BNS and the absence of such may be associated with poorer prognosis $[3,8,11,12]$. As demonstrated by Hiemcke-Jiwa et al, when clinical suspicion remains high for BNS and there is concern for low tumor DNA concentration within the CSF, highly sensitive double droplet 
PCR (ddPCR) techniques may be used as an alternative for identifying MYD88 L265P mutations [11]. In addition, testing for immunoglobulin gene rearrangements can help establish whether or not there are monoclonal heavy and light chain gene rearrangements in lymphoplasmacytic cells identified from the CSF, further augmenting the diagnostic workup of BNS [3]. Of note, the presence of these genetic mutations in either biopsy or CSF analysis is not necessarily specific for BNS as it can be found in other forms of primary CNS lymphoma $[3,8,11]$. Regardless of CSF results, biopsy of cerebral or meningeal lesions remains the gold stand for diagnosis of BNS with pathological evidence of lymphoplasmacytic lymphoma and immunochemical demonstration of monotypic B cells expressing antigens such as CD19, CD20, CD79a, and CD79b [3].

Given the paucity of BNS cases, there remains a lack of universal guidelines pertaining to the management of this disease process. Although there is no standardized approach for treatment, therapy should begin with identifying individuals who are symptomatic from their disease and requires antineoplastic agents that have the ability to penetrate the blood-brain barrier or agents available for intrathecal administration [3,13,21]. Treating those who are asymptomatic is felt to be contraindicated since the aim of treatment is not curative and tailored more towards reversing symptoms and inducing long term progression free survival $[3,13]$. Previously, systemic chemotherapy with purine nucleoside analogs such as cytarabine, fludarabine, and bendamustine, known to be effective at treating other lymphoproliferative disorders such as WM, showed promise as therapy for the treatment of BNS [14-16]. High-dose methotrexate and rituximab have been effective for the treatment of BNS as well, but only in combination with one another, as monotherapy with either of these agents has proven to be ineffective [13,14]. Rituximab itself is believed to have poor penetrance of the blood-brain barrier, thus contributing to its modest effect. In recent years, the oral Bruton tyrosine kinase (BTK) inhibitor ibrutinib has demonstrated promising results for the treatment of BNS, with it already having proven to be effective as therapy for WM [17-19]. The CNS penetrance of ibrutinib has been well-established in mouse models in addition to individual cases assessing the concentrations of active metabolite, PCI-45227, between synchronous measurements of plasma and CSF [18]. In a multicenter study conducted by Castillo et al. involving 28 patients with BNS treated with ibrutinib, it was found that $86 \%$ of patients had improvement of their associated symptoms with $83 \%$ of cases demonstrating tumor response on brain MRI [17].

Additional potentially targetable mutations have been identified throughout the past decade and thus offer further therapeutic alternatives for the treatment of WM and BNS. WHIM (warts, hypogammaglobulinemia, infections, and myelokathexis) syndrome caused by heterozygous mutations within the CXCR4 gene is observed in the pediatric population and characterized by chronic noncyclic neutropenia [24]. Although MYD88 L265P mutation is the most common somatic mutation in WM, this is followed closely by CXCR4 WHIM-like frameshift and nonsense mutations [22,23]. Plerixafor is an FDA-approved CXCR4 partial agonist and allosteric antagonist of CXCR7 which has been studied for clinical efficacy and safety in treatment of WHIM syndrome $[26,27]$. In vitro studies have demonstrated that ibrutinib resistance can be potentially reversed by CXCR4 inhibition, yet MYD88 inhibition superseded the survival benefits provided by CXCR4 frameshift mutations [25]. Therefore, it is theorized that plerixafor may have potential use in reversing ibrutinib failure in patients with WM and its efficacy is currently being evaluated within clinical trials. CXCL12 is another molecule that has been identified as an activator of AKT 1 and MPK1 pathways which helps confer the ability of malignant WM cells to resist ibrutinib-triggered apoptosis through mechanisms that increase frameshift and nonsense mutations [27]. Through continued research of molecular alterations leading to the development of WM and BNS, as well as genetic pathways contributing to resistance of targeted therapy, we can expect to see further improvement in both progression free and overall survival in this patient population.

As illustrated in our patient's case, switching from ibrutinib to rituximab had short-lived efficacy and soon led to worsening serum viscosity and IgM gammopathy. It is possible that she had already developed BNS at the time of disease progression which resulted in an inadequate response to rituximab monotherapy because of its poor blood-brain barrier permeability. Resuming therapy with ibrutinib resulted in significant improvements of her lab values and symptoms. However, despite continued treatment and relatively stable disease the patient developed BNS, raising concern given ibrutinib's proven efficacy for the treatment of BNS. Once systemic therapy was combined with WBRT there was noted improvement in both the patient's 
neurological symptoms as well as extent of disease evident on repeat imaging. Our case illustrates the importance of recognizing the early signs and symptoms of BNS within those who have a history of WM, regardless of their current and past treatment regimens. Diagnostic evaluation should be thorough and include biopsy of intracranial lesions when the suspicion remains high for BNS, as this remains the gold standard of practice. Early recognition of BNS can ultimately lead to more prompt treatment and rapid improvement of symptom burden, extended progression free survival, and overall improved quality of life in this scarce patient population.

\section{Conflicts of interest}

The authors of this manuscript declare no conflict of interest

\section{Author Contribution}

Lukas Delasos contributed to the preparation and presentation of the published work through creation of the initial draft, review of literature, and development of the finalized manuscript. Deep Phachu and Nishka Shetty contributed to critical review, commentary, and revision of the manuscript. Melissa Sepulveda-Ramos contributed to the pathological images and analysis. James Vredenburgh contributed to critical review and final revisions to the manuscript.

\section{References:}

1. Castillo, Jorge J., and Steven P. Treon. "Initial Evaluation of the Patient with Waldenström Macroglobulinemia." Hematology/Oncology Clinics of North America , vol. 32, no. 5, 2018, pp. 811-820., doi:10.1016/j.hoc.2018.05.008.

2. Castillo, Jorge J., et al. "Recommendations for the Diagnosis and Initial Evaluation of Patients with Waldenström Macroglobulinaemia: A Task Force from the 8th International Workshop on Waldenström Macroglobulinaemia." British Journal of Haematology , vol. 175, no. 1, May 2016, pp. 77-86., doi:10.1111/bjh.14196.

3. Minnema, Monique C., et al. "Guideline for the Diagnosis, Treatment and Response Criteria for Bing-Neel Syndrome." Haematologica , vol. 102, no. 1, June 2016, pp. 43-51., doi:10.3324/haematol.2016.147728.

4. Swerdlow, Steven H., et al. "The 2016 Revision of the World Health Organization Classification of Lymphoid Neoplasms." Blood , vol. 127, no. 20, 2016, pp. 2375-2390., doi:10.1182/blood-2016-01643569.

5. Arjunan, Ananth, and Hema Rai. "Central Nervous System Involvement by Waldenstrom Macroglobulinemia: A Case Report of the Bing-Neel Syndrome." Case Reports in Hematology , vol. 2019, 2019, pp. 1-3., doi:10.1155/2019/4075960.

6. Kulkarni, Tushar, et al. "Clinical Characteristics and Treatment Outcome Of CNS Involvement (BingNeel Syndrome) In Waldenstrom's Macroglobulinemia." Blood , vol. 122, no. 21, 2013, pp. 5090-5090., doi:10.1182/blood.v122.21.5090.5090.

7. Grainger, Brian T., and Samar Issa. "Bing-Neel Syndrome Presenting as Isolated CNS Lymphoplasmacytic Lymphoma: A Case Report and Review of the Literature." Journal of Clinical Neuroscience , vol. 71, 2020, pp. 277-280., doi:10.1016/j.jocn.2019.11.013.

8. Kopinska, Anna J., et al. "Bing-Neel Syndrome with Detectable MYD88 L265P Gene Mutation as a Late Relapse Following Autologous Hematopoietic Stem Cell Transplantation for Waldenström's Macroglobulinemia." Turkish Journal of Hematology , Nov. 2017, pp. 186-187., doi:10.4274/tjh.2016.0452.

9. Kim, Ho-Jung, et al. "Brain Magnetic Resolution Imaging to Diagnose Bing-Neel Syndrome." Journal of Korean Neurosurgical Society, vol. 46, no. 6, 2009, p. 588., doi:10.3340/jkns.2009.46.6.588.

10. Matsuda, Sakino, et al. "An Autopsy Case of Bing-Neel Syndrome: Discrepancy between the Radiological and Pathological Findings." Internal Medicine , vol. 58, no. 13, Jan. 2019, pp. 1947-1951., doi:10.2169/internalmedicine.1907-18.

11. Hiemcke-Jiwa, Laura S., et al. "The Use of Droplet Digital PCR in Liquid Biopsies: A Highly Sensitive Technique for MYD88 p.(L265P) Detection in Cerebrospinal Fluid." Hematological Oncology , vol. 36, 
no. 2, June 2017, pp. 429-435., doi:10.1002/hon.2489.

12. Poulain, Stéphanie, et al. "MYD88 L265P Mutation Contributes to the Diagnosis of Bing Neel Syndrome." British Journal of Haematology, U.S. National Library of Medicine, Nov. 2014, www.ncbi.nlm.nih.gov/pubmed/25160558/.

13. Castillo JJ et al. "How we manage Bing-Neel syndrome". Br J Haematol . 2019;187(3):277-285. doi:10.1111/bjh.16167

14. Vos, Josephine M.i., et al. "Effective Treatment of Bing-Neel Syndrome with Oral Fludarabine: a Case Series of Four Consecutive Patients." British Journal of Haematology, vol. 172, no. 3, May 2015, pp. 461-464., doi:10.1111/bjh.13483.

15. Delgado, Julio, et al. "Radiation Therapy and Combination of Cladribine, Cyclophosphamide, and Prednisone as Treatment of Bing-Neel Syndrome: Case Report and Review of the Literature." American Journal of Hematology, vol. 69, no. 2, 2002, pp. 127-131., doi:10.1002/ajh.10023.

16. Varettoni, Marzia, et al. "Successful Treatment with Rituximab and Bendamustine in a Patient with Newly Diagnosed Waldenströms Macroglobulinemia Complicated by Bing-Neel Syndrome." American Journal of Hematology, vol. 90, no. 8, 2015, doi:10.1002/ajh.24059.

17. Castillo, J., et al. "Ibrutinib For The Treatment Of Bing-Neel Syndrome: A Retrospective, Multicenter Study." Hematological Oncology, vol. 37, 2019, pp. 183-184., doi:10.1002/hon.140_2629.

18. Mason, Christopher, et al. "Ibrutinib Penetrates the Blood Brain Barrier and Shows Efficacy in the Therapy of Bing Neel Syndrome." British Journal of Haematology, vol. 179, no. 2, 2016, pp. 339-341., doi:10.1111/bjh.14218.

19. Hashmi, Hamza, et al. "Rare Case of Bing-Neel Syndrome Treated Successfully with Ibrutinib." BMJ Case Reports, vol. 12, no. 6, 2019, doi:10.1136/bcr-2019-230067.

20. Wong, Jonathan, et al. "Efficacy of Zanubrutinib in the Treatment of Bing-Neel Syndrome." HemaSphere, 2018, p. 1., doi:10.1097/hs9.0000000000000155.

21. Gavriatopoulou, Maria, et al. "Treatment of Bing-Neel Syndrome with First Line Sequential Chemoimmunotherapy." Medicine, vol. 98, no. 44, 2019, doi:10.1097/md.0000000000017794.

22. Fitsiori, A et al. "Imaging spectrum of Bing-Neel syndrome: how can a radiologist recognise this rare neurological complication of Waldenström's macroglobulinemia?". Eur Radiol 29, pp. 102-114 (2019). https://doi.org/10.1007/s00330-018-5543-7.

23. Treon, Steven P., et al. "MYD88 L265P Somatic Mutation in Waldenström's Macroglobulinemia". $N$ Engl J Med 2012. 367:826-833. DOI: 10.1056/NEJMoa1200710

24. Ansell, S. et al. "Activation of TAK1 by MYD88 L265P drives malignant B-cell Growth in nonHodgkin lymphoma." Blood Cancer Journal 4, e183 (2014). https://doi.org/10.1038/bcj.2014.4

25. Dotta L., et al. "Clinical And Genetic Features Of Warts, Hypogammaglobulinemia, Infections And Myelokathexis (WHIM) Syndrome." Curr Mol Med. 2011 Jun: 11(4):317-25.

26. Cao, Y. et al, "CXCR4 WHIM-like frameshift and nonsense mutations promote ibrutinib resistance but do not supplant MYD88 L265P-directed survival signalling in Waldenstrom macroglobulinaemia cells". Br J Haematol, 168: 701-707. doi:10.1111/bjh.13200

27. Kalatsakaya, I. et al, "AMD3100 Is a CXCR7 Ligand with Allosteric Agonist Properties." Molecular Pharmacology May 1, 2009, 75 (5) pp. 1240-1247; DOI: https://doi.org/10.1124/mol.108.053389

28. McDermott, David H., et al. "Plerixafor For The Treatment Of WHIM Syndrome."

N Engl J Med 2019; 380:163-170.DOI: 10.1056/NEJMoa1808575.

\section{Hosted file}

BNS figure 1.docx available at https://authorea.com/users/321591/articles/450809-developmentof-bing-neel-syndrome-despite-ibrutinib-therapy-for-waldenstrom-macroglobulinemia-nextsteps-in-management-and-review-of-future-directions

\section{Hosted file}

BNS Figure 2.docx available at https://authorea.com/users/321591/articles/450809-developmentof-bing-neel-syndrome-despite-ibrutinib-therapy-for-waldenstrom-macroglobulinemia-next- 
steps-in-management-and-review-of-future-directions

\section{Hosted file}

BNS Figure 3.docx available at https://authorea.com/users/321591/articles/450809-developmentof-bing-neel-syndrome-despite-ibrutinib-therapy-for-waldenstrom-macroglobulinemia-nextsteps-in-management-and-review-of-future-directions 